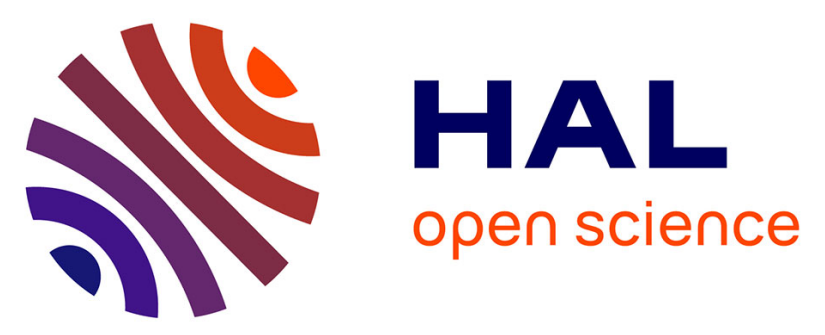

\title{
Robust Method of Stabilization of Optical Feedback Regime by using Adaptive Optics for a Self-Mixing Micro-Interferometer Laser Displacement Sensor
}

Olivier Bernal, Usman Zabit, Thierry Bosch

\section{- To cite this version:}

Olivier Bernal, Usman Zabit, Thierry Bosch. Robust Method of Stabilization of Optical Feedback Regime by using Adaptive Optics for a Self-Mixing Micro-Interferometer Laser Displacement Sensor. IEEE Journal of Selected Topics in Quantum Electronics, 2015, 21 (4), pp.336-343. 10.1109/JSTQE.2014.2381494 . hal-01262360

\section{HAL Id: hal-01262360 \\ https://hal.science/hal-01262360}

Submitted on 27 Jan 2016

HAL is a multi-disciplinary open access archive for the deposit and dissemination of scientific research documents, whether they are published or not. The documents may come from teaching and research institutions in France or abroad, or from public or private research centers.
L'archive ouverte pluridisciplinaire HAL, est destinée au dépôt et à la diffusion de documents scientifiques de niveau recherche, publiés ou non, émanant des établissements d'enseignement et de recherche français ou étrangers, des laboratoires publics ou privés. 


\title{
Robust Method of Stabilization of Optical Feedback Regime by using Adaptive Optics for a Self-Mixing Micro-Interferometer Laser Displacement Sensor
}

\author{
Olivier D. Bernal, Usman Zabit, and Thierry Bosch \\ http://dx.doi.org/10.1109/JSTQE.2014.2381494 (C) 2015 IEEE \\ January 27, 2016
}

\begin{abstract}
A self-mixing (SM) micro-interferometer laser displacement sensor coupled with an adaptive liquid lens (ALL) system is proposed and implemented. This has been made possible by a new method of real-time estimation of the optical feedback coupling factor $C$. It is shown that such an estimation of $C$ combined with an appropriate amplification of the SM signal Gain allows the ALL system to seek and maintain the SM signal in the moderate optical feedback regime in spite of variations in the optical feedback. The ALL system thus enables robust real-time displacement sensing in an unmanned autonomous manner. The implemented system has provided measurement precision better than $90 \mathrm{~nm}$ for different target surfaces and distances. The paper also investigates the impact of the weighting attributed to $C$ and Gain on the retrieved displacement precision. As this autofocus is presently only performed once during the sensor initialization, so maximum displacement span after achieving optical feedback regime locking has also been investigated and tabulated. This proof of concept, thus paves the way for the deployment of autonomous SM sensors.
\end{abstract}

\section{Introduction}

Self-mixing effect [1,2] occurs in a laser when part of a beam back-scattered from a remote target is coupled back into the active laser cavity, thereby causing interference. The laser diode (LD) then simultaneously acts as a 1)

doi: http://dx.doi.org/10.1109/ICSENS.2015.7370375 (c) 2015 IEEE 
(C) 2015 IEEE Personal use of this material is permitted. Permission from IEEE must be obtained for all other users, including reprinting/ republishing this material for advertising or promotional purposes, creating new collective works for resale or redistribution to servers or lists, or reuse of any copyrighted components of this work in other works

micro-interferometer, 2) a laser source, as well as 3) a detector (either by using LD junction voltage or the photodiode contained in the LD package). This then allows a simple, compact, self-aligned and cost effective sensor design. However, such an apparent simplicity comes at a cost of complex nature of these interferometric signals arising due to optical feedback inside the active laser cavity. So, a major challenge in the design of a robust realtime SM sensor is posed by this rich variety of SM signals as significant variations in the remote target surface reflectivity $R_{t}$ and the distance to the remote target $D$ cannot be avoided.

SM signal waveforms have been reported to depend on the $C$ factor as well as the LD linewidth enhancement factor $\alpha$ [1,3,4]. The $C$ factor [5] is usually used to distinguish between three main optical feedback regimes [1]:

- $0.1<C<1$ : weak optical feedback regime with sinusoidal or asymmetric SMI fringes devoid of sharp discontinuities [6].

- $1<C<4.6$ : moderate optical feedback regime with sawtooth-like SMI fringes exhibiting hysteresis [3].

- $4.6<C$ : strong optical feedback regime that ultimately leads to a chaotic SM signal [1,4, 7].

Even though different algorithms exist to process different feedback regime signals [6, 8-11], to the best of our knowledge, no universal algorithm exists to process all of the SM regimes. Due to absence of a universal algorithm, it becomes advisable to somehow maintain a stable feedback regime. Consequently, the moderate optical feedback regime is often sought for displacement sensing as its supposedly straight-forward signal shape [3] leads to simplified signal processing. One can then use half-fringe phase locking [10] or employ various phase unwrapping methods [9, 11,12]. In this paper, SM signals have been processed in real-time by using the Consecutive Samples based Unwrapping (CSU) method [1] having a measurement precision of $\lambda / 8$ where $\lambda$ is the LD wavelength. Note that as mentioned in [13, such precision can only be achieved if:

- The signal-to-noise ratio (SNR) is high enough to allow correct fringe detections.

- The $C$ factor of the moderate optical feedback regime SM signal is within appropriate range.

It will be shown that our proposed technique satisfies both conditions.

doi: http://dx.doi.org/10.1109/ICSENS.2015.7370375 (c) 2015 IEEE 
So, it is easy to observe that for such SM based displacement measurement methods, it is necessary to maintain the SM signal in a known, stable moderate optical feedback regime, especially when $R_{t}$ and $D$ may vary. This is usually achieved by a careful manual adjustment of optical feedback level, [10,11] since the $C$ factor can be modified by manually changing the distance between the LD and its collimating lens. However, this procedure is both cumbersome and non-trivial for unskilled users. Furthermore, it cannot obviously lead to automatic real-time sensing.

To get rid of such constraints, previous research demonstrated that a liquid lens can replace any mechanical lens positioning [14]. It was then shown that liquid lens increases the robustness of a SM sensor for a speckle affected SM signal [15]. The algorithm of [15] modifies the focal length of liquid lens $\left(f_{L L}\right)$ by relying only on the SNR of the affected SM signal. Another approach based on a mechanical pickup, similar to the one included in all CD players, is demonstrated in [8] whose autofocus system also calculates the $C$ value. However, as its value is proportional to the amplitude of the SM fringes, it is calculated only for the weak optical feedback regime. Similarly, another method of computing $C<1$ uses time durations of increasing and decreasing semiperiods of SM signals [16]. In addition, such an estimation can only be obtained either in case of a ramp-like (linear) target displacement or by linearly modulating the laser wavelength. Therefore, this method might not be convenient for arbitrary target displacement. In contrast, our proposed method of estimation of $C$, remains valid over almost all the moderate optical feedback regime range and does not require high computational resources. Furthermore, our novel autonomous optical feedback regime locking is achieved not just by tracking the SNR but is in fact based on a real-time estimation of $C$ in addition to a real-time assessment of amplification needs of SM signal amplitude $A_{S M}$ that is used to perform SM signal normalization. An autofocus system then uses both of these parameters to control an adaptive optics element (a voltage controlled liquid lens) in a feedback loop. In this way, displacement reconstruction can be efficiently achieved by the CSU algorithm as the obtained SM signal after autofocus belongs to the desired optical feedback regime. Note that it is an aim of this paper to demonstrate that both $C$ and $A_{S M}$ are necessary for robust optical feedback regime locking for a wide variety of target surfaces and sensor-target distances.

Fig. 1 gives an overview of the proposed adaptive liquid lens (ALL) based autofocus system, which has been implemented on and integrated to a previous real-time SM vibrometer prototype employing CSU [11].

The central idea of our proposed approach consists thus in controlling $f_{L L}$ by a feedback loop (that uses both the estimated $C$ value and the amplitude

doi: http://dx.doi.org/10.1109/ICSENS.2015.7370375 (C) 2015 IEEE 


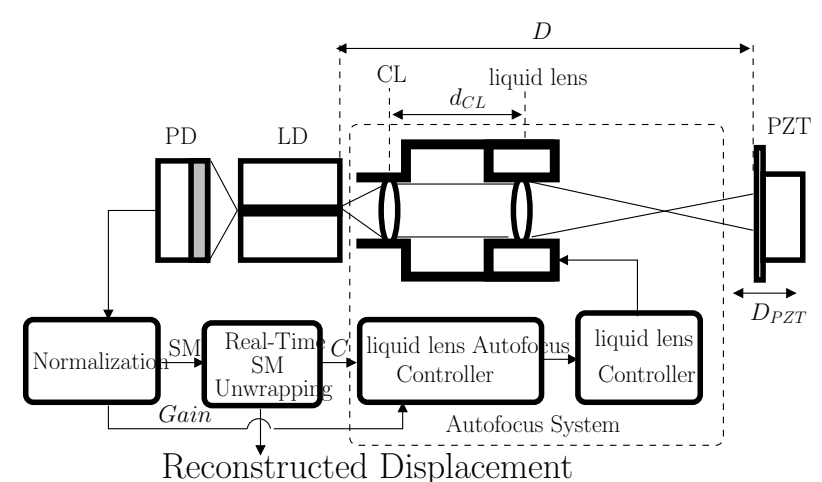

Figure 1: Schematic diagram of the adaptive liquid lens based real-time SM displacement sensor.

$A_{S M}$ of the acquired real-time SM signal) so as to ensure that the LD based micro-interferometer is operating in the moderate optical feedback regime.

The paper is organized as follows. After a short introduction of the main characteristics of SM signals, we shall propose a method to estimate the $C$ factor in real-time. From this analysis, we shall derive our proposed autofocus algorithm. Finally, experimental validation of the proposed autofocus technique is presented followed by a discussion and conclusion.

\section{Theory of Self-Mixing}

The theory of SM interferometry has been described by various authors [17][18] and is briefly summarized below. Let $D(t)$ represent the instantaneous distance between the LD driven by a constant injection current and a remote surface that back-scatters a small amount of optical power back into the LD cavity.

When this optical feedback phenomenon occurs, the laser wavelength is no longer the constant $\lambda_{0}$ but is slightly modified and becomes a function of time $\lambda_{F}(t)$ varying with $D(t)$. The wavelength fluctuations can be found by solving the phase equation [19]:

$$
\Phi_{0}(t)=\Phi_{F}(t)+C \sin \left[\Phi_{F}(t)+\operatorname{atan}(\alpha)\right]
$$

where $\Phi_{F}$ and $\Phi_{0}$ represent two phase signals (subject to feedback and under free running conditions, respectively).

The value of $\Phi_{F}(t)$ can be extracted from the optical output power (OOP) of the laser diode $P(t)$ using:

$$
P(t)=P_{0}\left\{1+m \cos \left[\Phi_{F}(t)\right]\right\},
$$

doi: http://dx.doi.org/10.1109/ICSENS.2015.7370375 (C) 2015 IEEE 
(C) 2015 IEEE Personal use of this material is permitted. Permission from IEEE must be obtained for all other users, including reprinting/ republishing this material for advertising or promotional purposes, creating new collective works for resale or redistribution to servers or lists, or reuse of any copyrighted components of this work in other works

where $P_{0}$ is the power emitted by the free running state laser diode and $m$ a modulation index. By using $\Phi_{F}, \Phi_{0}(t)$ can first be retrieved using the nonlinear equation eq. 1 and then the vibration displacement $d(t)$ as shown in [12].

Further, the $C$ factor ( eq. 11) depends on some LD features but also on both $D$ and $R_{t}$ [8]. It can be expressed as in [16]:

$$
C=\frac{\tau_{D}}{\tau_{L}} \gamma \sqrt{1+\alpha^{2}} \kappa_{e x t}
$$

where $\tau_{L}$ and $\tau_{D}$ are the round trip times in the internal and external cavities respectively, $\gamma$ the coupling efficiency and $\kappa_{e x t}$ depends linearly on the surface reflectivity of the target.

\section{$3 \quad C$ factor real-time estimation method}

From the previous section, it can be deduced that the $C$ factor value needs to be assessed so that different SM optical feedback regimes can be distinguished and the SM sensor can potentially be locked to the moderate optical feedback regime. For this purpose, different methods can be used to estimate the $C$ factor with varying accuracy, such as [5, 12, 20]. However, neither the optical feedback regime locking nor the employed SM unwrapping method (CSU) requires an estimation of $C$ value accurate up to 1 decimal place . Actually, using the CSU [13], the target displacement is obtained by adding the unwrapped discontinuities (corresponding to a $\lambda / 2$ displacemnt each) to the instantaneous SM signal (see Fig. 2). So, the accuracy (provided at large computational cost) of approaches such as [5, 12, 20] is not necessary. Nevertheless, due to the inherent simplicity of the CSU, the reconstruction is subject to errors directly related to both the size and shape of SM fringes (Fig. 2). For instance, Fig. 3 shows that the CSU provides minimal RMS error in displacement reconstruction if $C \in[1.5,2.5]$. Note that similar results can be obtained for different displacement amplitudes. As a result, in order to achieve better measurement precision with the CSU, $C$ should not just be maintained in the moderate feedback regime but more specifically to within $[1.5,2.5]$.

In this regard, progress can be made by observing the SM signal itself as its shape depends on $C$. By extracting some key features from SM signals, $C$ value estimation can be achieved. One such striking feature of SM signal in moderate optical feedback regime is its sawtooth like appearance with prominent discontinuities. For a SM signal with good SNR, such discontinuities can be easily detected and a method is proposed below for estimating the $C$ value, by measuring the amplitude of these discontinuities.

doi: http://dx.doi.org/10.1109/ICSENS.2015.7370375 (c) 2015 IEEE 


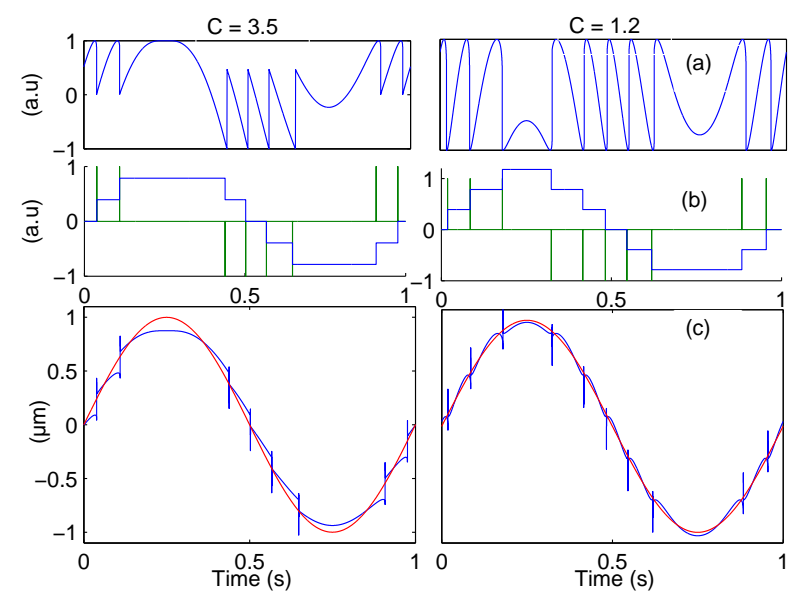

Figure 2: Displacement Retrieval through Consecutive-samples based Unwrapping. (a) Normalized SM signal, (b) fringe discontinuity detection (green) and staircase signal representing the fringe count (blue) and (c) the retrieved displacement obtained by the addition of SM signal and staircase (blue) in comparison with the target displacement (red).

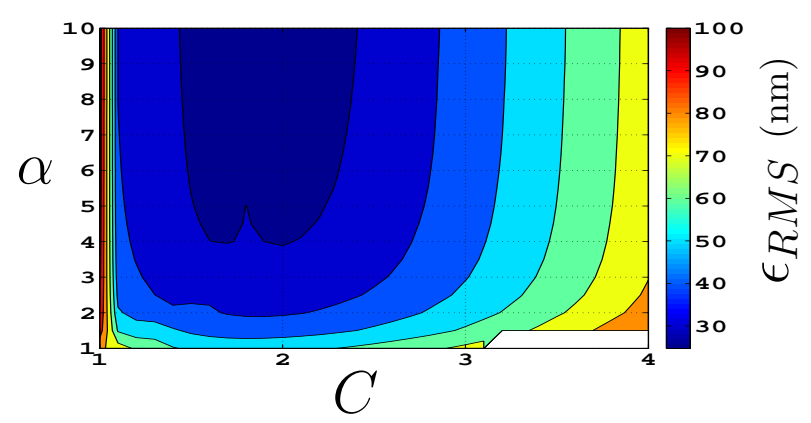

Figure 3: Simulated displacement reconstruction RMS error $\epsilon_{R M S}$ of the CSU algorithm versus $C$ and $\alpha$ for a $2.5 \mu \mathrm{m}$ sinusoidal displacement.

As suggested by eq. 2, such discontinuities are due to $\Phi_{F}$ hopping phenomenon. Consequently, the relation between phase discontinuity and $C$ will be first derived. For $C>1$, these discontinuities can be numerically observed by solving the phase equation eq. 11 [17. It can be shown that such phase discontinuities occur whenever the function $\Phi_{F}(t)=F\left[\Phi_{0}(t), C, \alpha\right]$ has infinite slopes [4] (see Fig. 44):

$$
\begin{aligned}
& \Phi_{F, R}=k \pi-\arctan (\alpha)+\beta \\
& \Phi_{F, F}=(k+2) \pi-\arctan (\alpha)-\beta
\end{aligned}
$$

where $k$ is an even integer and $\beta=\arccos -1 / C$. These discontinuities can doi: http://dx.doi.org/10.1109/ICSENS.2015.7370375 (C) 2015 IEEE 


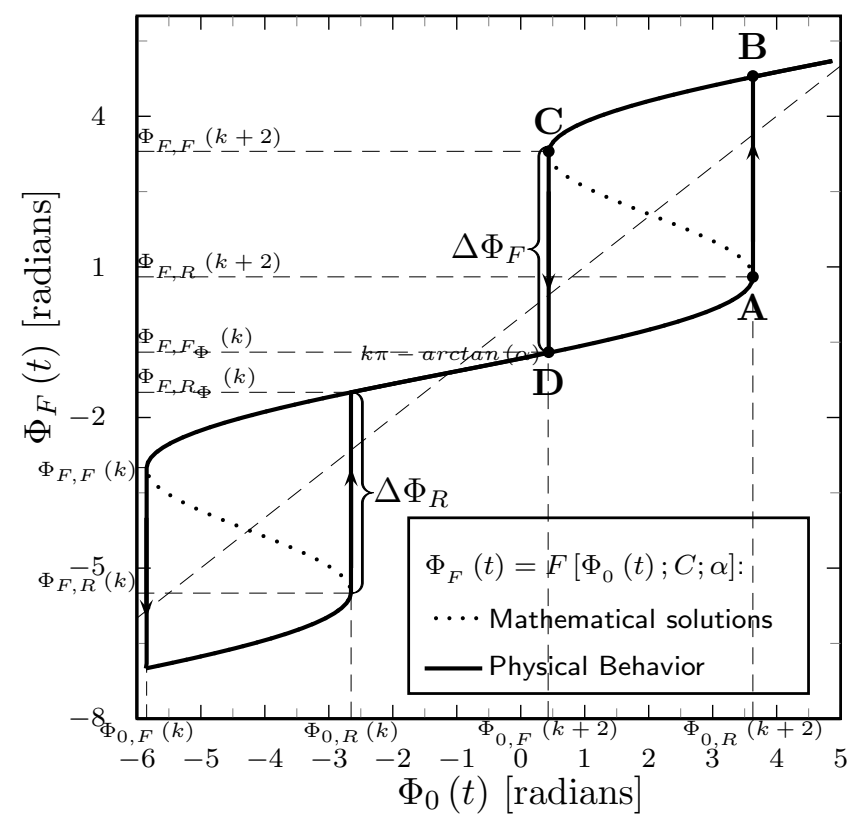

Figure 4: Transfer function $\Phi_{F}(t)=\mathrm{F}\left[\Phi_{0}(t) ; C, \alpha\right]$ with hysteresis $(C=3$ and $\alpha=2)$ and $k$ is an even integer.

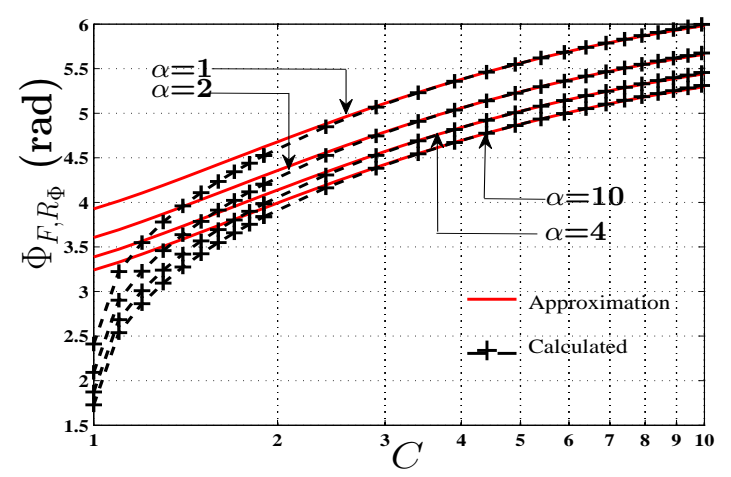

Figure 5: Numerically calculated (black dashed line) and analytically approximated (red line) $\Phi_{F, R_{\Phi}}$.

be either rising $\Delta \Phi_{R}$ or falling $\Delta \Phi_{F}$ depending on the evolution of $\Phi_{F}(t)$. After the phase rising (falling) discontinuity, the phase value $\Phi_{F, R_{\Phi}}\left(\Phi_{F, F_{\Phi}}\right)$ can be estimated numerically (Fig. 4). Here, we focus only on rising phase discontinuities as it contains enough information to roughly estimate $C$. A fair approximation of $\Phi_{F, R_{\Phi}}($ error $<10 \%)$ can nevertheless be analytically calculated for $C>1.5$. Such an approximation can be obtained by equaling $\Phi_{0}\left(\Phi_{F, R}\right)$ and the tangent of the curve described by eq. 1] at $\Phi_{F}=\Phi_{0}$ [4].

doi: http://dx.doi.org/10.1109/ICSENS.2015.7370375 (c) 2015 IEEE 
(C) 2015 IEEE Personal use of this material is permitted. Permission from IEEE must be obtained for all other users, including reprinting/ republishing this material for advertising or promotional purposes, creating new collective works for resale or redistribution to servers or lists, or reuse of any copyrighted components of this work in other works

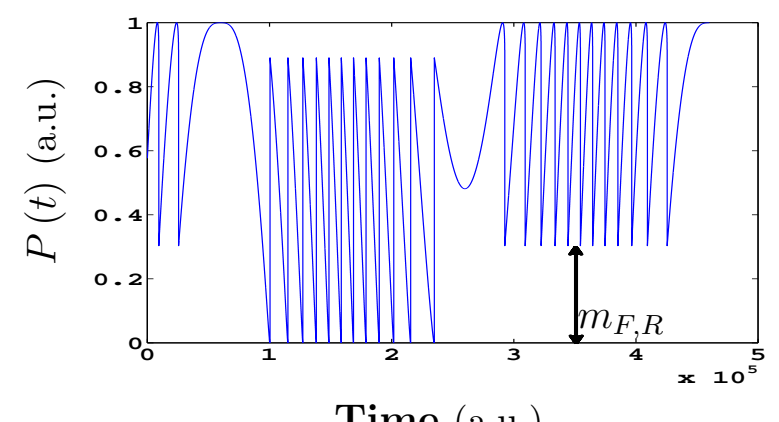

Figure 6: Simulated normalized SM signal for $C=2.4$ and $\alpha=4$.

This approximation is compared to the numerically calculated one in Fig. 5 and can be expressed as follows:

$$
\Phi_{F, R_{\Phi}}[2 \pi] \approx-\arctan (\alpha)+\frac{\beta+C(\sin (\beta)+2 \pi)}{1+C}
$$

Using the partial derivative of the $\Phi_{F, R_{\Phi}}$ approximation with respect to $C$, it can be shown that $\Phi_{F, R_{\Phi}}$ is monotonically increasing with $C$ (Fig. [5) . Further, by using results from [3], it can be shown that for $C>1.5, \Phi_{F, R_{\Phi}}>$ $\pi$. This last result implies that the local minimum height $m_{F, R}$ (see Fig. 6) of the measured corresponding fringe (rising discontinuity) in the SM signal, which corresponds to $\Phi_{F, R_{\Phi}}$, increases with increasing value of $C$, thereby providing a method of estimating $C$ if the amplitude of the measured SM signal is kept constant for a given $\alpha$ (see Fig. 7). It then explains why the normalization step is important: 1) to maximize the SM signal , and 2) to ease the $C$ value extraction in real-time. The normalization procedure is described in the next section. However, note that as mentioned in [20], the normalization process cannot be usually performed correctly for one way linear displacement (the target moves in one direction only).

Note that for lower value of $C$, the bijection between the measured minimum local value after a rising phase discontinuity and $\Phi_{F, R_{\Phi}}$ (and thus $C$ ) is no more ensured [3]. Further, as highlighted by eq, 6 , the $\Phi_{F, R_{\Phi}}$ value also depends on $\alpha$. Note that a much more accurate algorithm for $C$ estimation could be designed and used based on the combination of $\Phi_{F, R_{\Phi}}$ and $\Phi_{F, F_{\Phi}}$ (value of the phase after the falling discontinuity) at the expense of more computational cost. However, such a complex algorithm has not been used as the lack of precise $C$ estimation has a minimal effect on both the CSU (see Fig. 3) and the autofocus algorithm for the desired precision $(\lambda / 8)$. Fig. 8 shows that the relative error $\Delta C / C$ of the proposed estimation of $C$ is less than $20 \%$ for $C \in[1.5,10]$. As expected, the approximation of $\Phi_{F, R_{\Phi}}$ is not

doi: http://dx.doi.org/10.1109/ICSENS.2015.7370375 (C) 2015 IEEE 


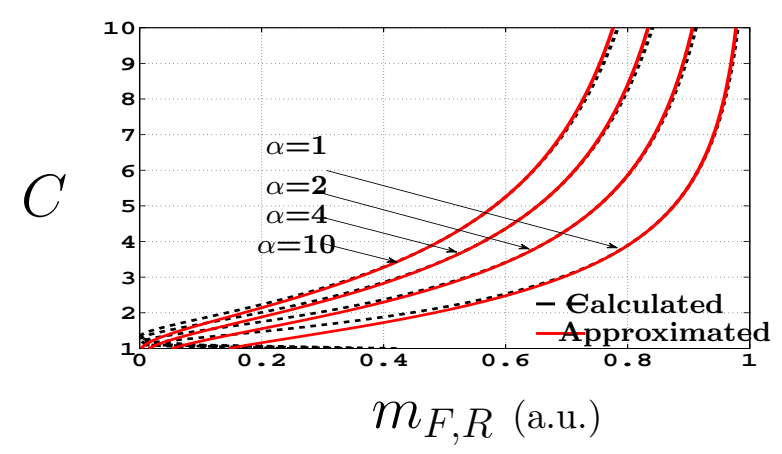

Figure 7: Numerically calculated (black dashed line) and analytically approximated (red line) $C$ versus the height of SM fringe minimum $m_{F, R}$.

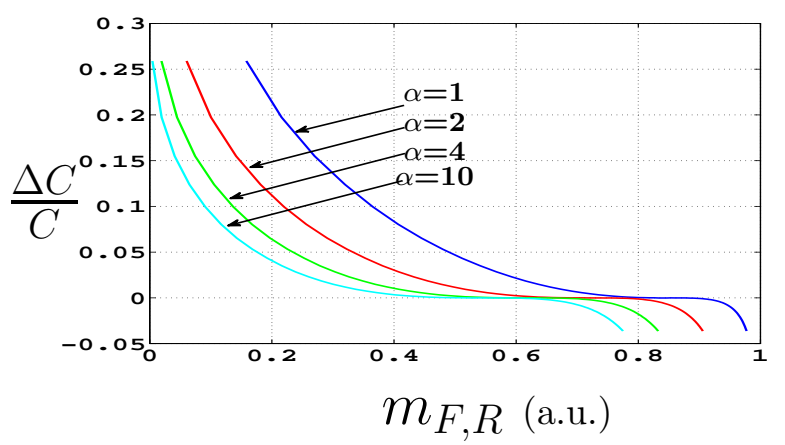

Figure 8: Numerically calculated relative error $\Delta C / C$ of the proposed estimation of $C$ versus the height of SM fringe minimum $m_{F, R}$.

very accurate for low $C$ value, which consequently leads to a lack of accuracy to estimate $C$ based on low $m_{F, R}$ values. Nevertheless, as previously mentioned, CSU can achieve higher precision for $C \in[1.5,2.5]$ which corresponds to $m_{F, R} \in[0.02 ; 0.6]$ for $\alpha \in[1 ; 10]$.

From the analysis of Fig. 7, a linear approximation can give better result for such a restricted $m_{F, R}$ range. With an aim of further simplifying the algorithm for a real-time implementation, only one linear approximation (calculated for $\alpha=4$ ) independent of $\alpha$ has been used. The relative error $\Delta C / C$ of the proposed linear estimation of $C$ is less than $20 \%$ for $C \in[1.5,2.5]$ except for $\alpha<2$. It demonstrates that the value of $\alpha$ does not critically affect the estimation of $C$ based on $m_{F, R}$.

Finally, Fig. 10 shows that if $C \in[1.5,2.5] \pm 20 \%$, the RMS displacement reconstruction error is still below $\lambda / 8$.

doi: http://dx.doi.org/10.1109/ICSENS.2015.7370375 (c) 2015 IEEE 


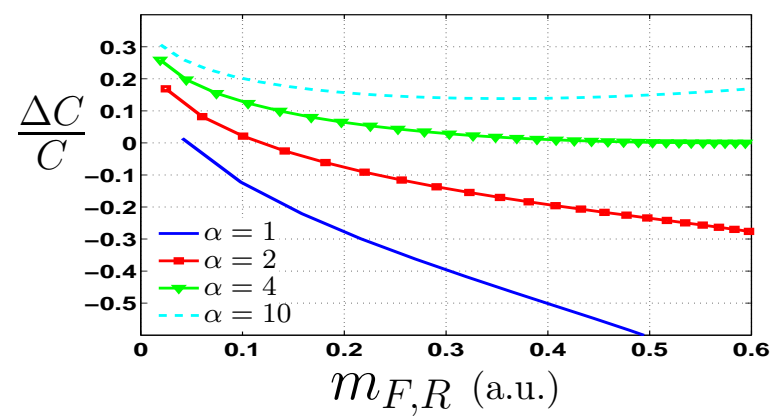

Figure 9: Numerically calculated relative error $\Delta C / C$ of the proposed linear estimation of $C$ versus the height of SM fringe minimum $m_{F, R}$.

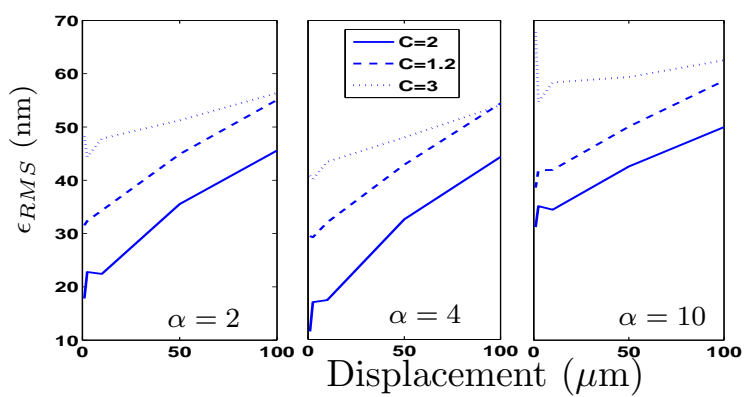

Figure 10: Numerically calculated RMS reconstruction error for a CSU optimal value of $C=2$ and two $C$ values out of the $[1.5,2.5]$ range by $20 \% C=1.2$ and $C=3$.

\section{Autofocus Algorithm}

Before presenting the autofocus algorithm, let's first describe how the amplitude of the SM signal $A_{S M}$ is processed since the approach used is also relevant for the real-time estimation of $C$ as mentioned in the previous section. In the present case, $A_{S M}$ has been processed by using a related parameter, denoted Gain $\left(\propto 1 / A_{S M}\right)$, which is obtained during the stage of SM normalization (see Fig. 1 and 11). The primary purpose of Gain is to ensure that the real-time amplitude of SM signal is normalized. Otherwise, lack of normalization causes errors in displacement reconstruction during CSU [13]. This is so because the CSU method can achieve a precision of $\lambda / 8$ only for a pre-defined amplitude of SM signal and appropriate $C$ value. In fact, the peak-to-peak amplitude of the fringes of the scaled SM signal must ideally match the step size chosen for SM unwrapping at an initial precision of $\lambda / 2$ [13]. Therefore, the scaling/normalization of the SM signal is necessary, and a closed-loop normalization circuit (based on SM peak detectors

doi: http://dx.doi.org/10.1109/ICSENS.2015.7370375 (c) 2015 IEEE 


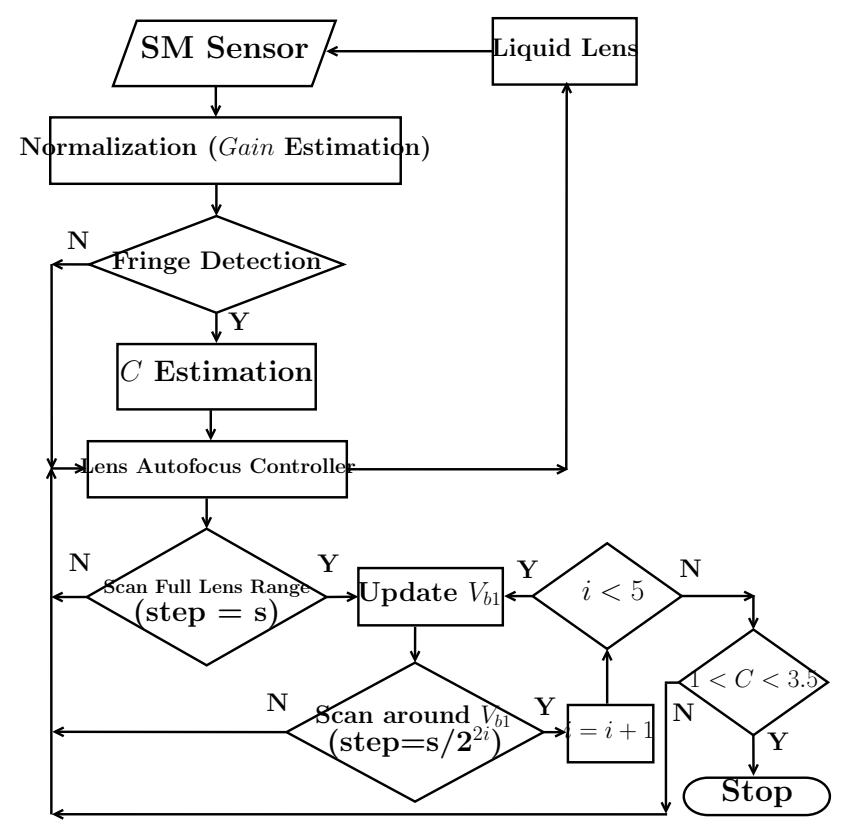

Figure 11: Flowchart of the ALL autofocus algorithm.

and a variable gain amplifier (VGA) such as the AD603) has been developed to maintain a $2.3 \mathrm{~V}_{\text {Peak-Peak }} \mathrm{SM}$ signal amplitude.

Now, this normalization feedback loop thus contains a piece of information (the Gain parameter) which is related to the SM SNR. Hence, it can be easily retrieved and fed to the autofocus system (see Fig. 11).

Let's now elaborate the algorithm of the preliminary autofocus (see Fig. 111) used for optical feedback regime locking. It is composed of five phases. In case of detection of SM fringes, the liquid lens Autofocus Controller analyzes the $C$ and Gain values (provided by ADuC7020 and AD603 respectively) and computes the output value of the cost function $w_{C} C+w_{G} / A_{S M}$ (implemented in the dsPic33F128GP802), where $w_{C}$ and $w_{G}$ represent the weights attributed to $C$ and Gain respectively. Thus, during the first scanning phase, the algorithm scans the full span range of liquid lens in a step-wise manner and computes the corresponding cost function at each step. At the same time, it keeps on updating the liquid lens voltage denoted as $V_{b 1}$ for which the minimum value of cost function has been found until that instant. It is the voltage which results in a SM signal with an acceptable value of $C$ as well as an $A_{S M}$ value ensuring a correct SM normalization. In addition, in order to ensure that the SM signal is maintained in the moderate feedback regime, the algorithm automatically discards SM signals with $m_{F, R}<0.03$ or $>0.6$ (as suggested by the bottom right decision block of Fig. 11). The next

doi: http://dx.doi.org/10.1109/ICSENS.2015.7370375 (c) 2015 IEEE 
(C) 2015 IEEE Personal use of this material is permitted. Permission from IEEE must be obtained for all other users, including reprinting/ republishing this material for advertising or promotional purposes, creating new collective works for resale or redistribution to servers or lists, or reuse of any copyrighted components of this work in other works

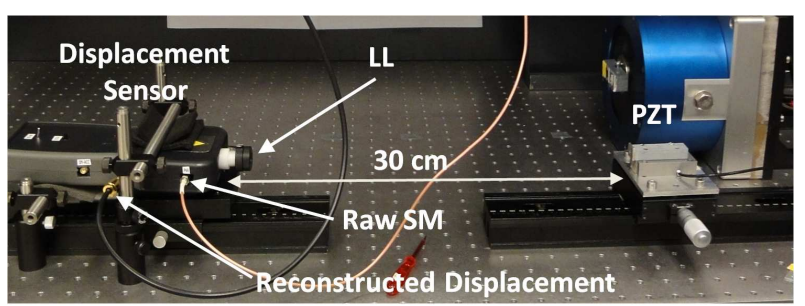

Figure 12: Experimental set-up of auto-adaptive liquid lens based system for autonomous Self-Mixing feedback regime locking (see media 1).

phases are similar, except that for each iterative phase, the scanning process is centered around the previously found $V_{b 1}$ with a narrower step and a span range equal to twice the former step size. Note that the liquid lens and the normalization loop need approximately $80 \mathrm{~ms}$ [21] and $120 \mathrm{~ms}$ respectively to stabilize. As a result, the autofocus is done within $30 \mathrm{~s}$ while adjustment around this position only requires $0.2 \mathrm{~s}$.

\section{$5 \quad$ Experimental Results}

Our experimental setup is described below (see Fig. 12). The deployed liquid lens is an ARTIC 39N0 liquid lens from Varioptic. This liquid lens has an optical power $\left(1 / f_{L L}\right)$ variation of -5 to 15 diopters. The optical casing containing the liquid lens and the collimation lens (CL) is placed in front of the LD (HL7851 emitting at $\lambda=785 \mathrm{~nm}$ ). The linewidth enhancement factor $\alpha$ was estimated to be approximately 3.6 using the method described in [22]. The CL collimates the laser beam onto the liquid lens which is placed at $d_{C L}=18 \mathrm{~mm}$. The target is the $\mathrm{P}-753.2$.CD piezoelectric transducer (PZT) from Physik Instrumente placed at a distance $D$ from the LD. It has a builtin capacitive feedback sensor with $2 \mathrm{~nm}$ precision that was used as a reference sensor for the PZT movement $\left(D_{P Z T}\right)$. The SM signal is picked-up by the built-in monitor Photo-Diode (PD) of the LD package. The CSU is executed by using AduC7020 of Analog Devices ${ }^{\circledR}$, whereas liquid lens Autofocus Controller is embedded on dsPic33F128GP802 of Microchip ${ }^{\circledR}$ [11.

The experimental SM signals obtained after autofocus for three different distances are shown in Fig. 13. It demonstrates the robustness of the autofocus system. On the other hand, Fig. 14 highlights the influence of the $w_{G} / w_{C}$ ratio on the SM signal $P(t)$ and its corresponding reconstructed displacement $D_{r}(t)$. It can be seen that changing the $w_{G} / w_{C}$ ratio changes the SM feedback regime, which in turn affects the measurement precision. Therefore, in order to fully characterize the ALL autofocus system, it has

doi: http://dx.doi.org/10.1109/ICSENS.2015.7370375 (C) 2015 IEEE 
(c) 2015 IEEE Personal use of this material is permitted. Permission from IEEE must be obtained for all other users, including reprinting/ republishing this material for advertising or promotional purposes, creating new collective works for resale or redistribution to servers or lists, or reuse of any copyrighted components of this work in other works

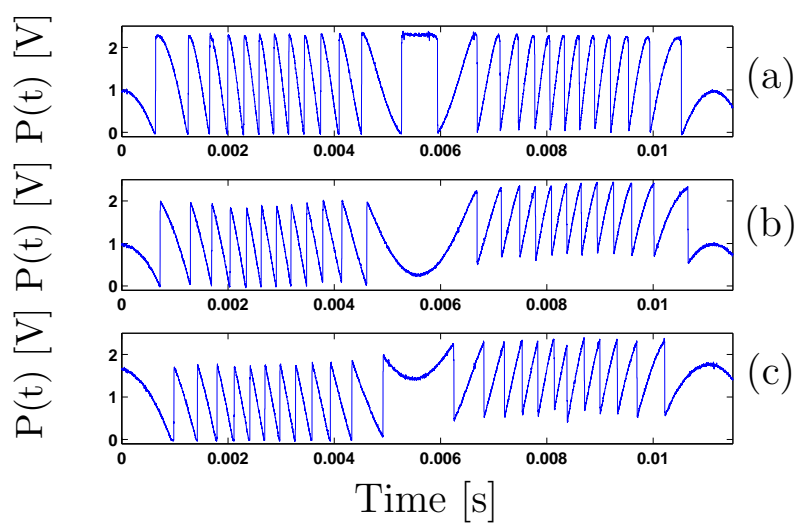

Figure 13: Measured normalized and auto-focused SM signal $\mathrm{P}(\mathrm{t})$ for a steel target (90 Hz vibration, $2.5 \mu \mathrm{m}$ amplitude) for $D=$ (a) $15 \mathrm{~cm}$, (b) $55 \mathrm{~cm}$ and (c) $90 \mathrm{~cm}$.

been deemed necessary to thoroughly analyze the dependency of the $w_{G} / w_{C}$ ratio on:

- the target surface characteristics

- the laser-target distance $D$

In addition, though the $C$ factor is estimated in real time, the autofocus implemented here is still only performed once. Consequently, it is necessary to assess the performance of ALL system in terms of maximum target displacement that can be measured with expected precision after the initial feedback regime locking.

\subsection{Target surface influence}

As mentioned in eq. 3, the $C$ factor directly depends on the target surface reflectivity. Now, we know that the autofocus algorithm aims to lock on to CSU compatible SM signals (having low $C$ and good SNR) by taking into account the weights attributed to both $C$ and Gain values. So, it can then be understood that the optimal $w_{G} / w_{C}$ ratio (resulting in the most compatible SM signal) strongly depends on the target surface reflectivity.

For instance, in the case of a highly reflective (diffusive) surface, the resulting $1 / A_{S M}$ may be low (high) while $C$ factor may be high (low). Consequently, for a highly reflective surface, better feedback regime locking can be achieved by increasing $w_{C}$ as compared to $w_{G}$ as in such a case, SM signals with low $C$ values can be more difficult to obtain, contrary to SM signals

doi: http://dx.doi.org/10.1109/ICSENS.2015.7370375 (c) 2015 IEEE 
(C) 2015 IEEE Personal use of this material is permitted. Permission from IEEE must be obtained for all other users, including reprinting/ republishing this material for advertising or promotional purposes, creating new collective works for resale or redistribution to servers or lists, or reuse of any copyrighted components of this work in other works

(a)

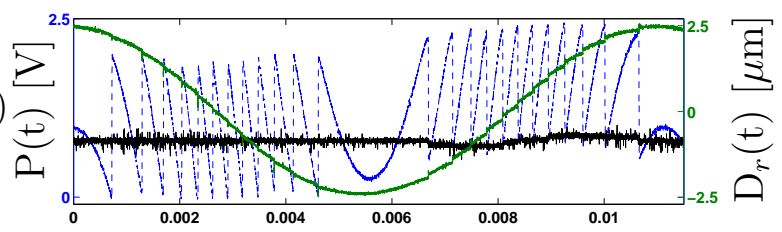

(b)

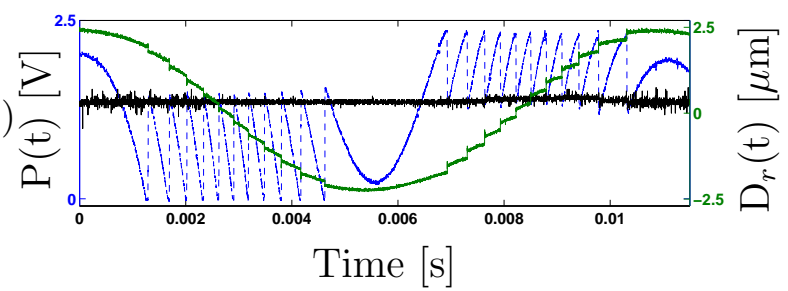

Figure 14: Measured normalized and auto-focused SM signal P(t) (blue), $D_{r}(\mathrm{t})$ (green) and $m_{F, R}$ estimation (black) for two different $w_{G} / w_{C}$ ratios: (a) 2 (see media 2) and (b) 4 (see media 3); for a $90 \mathrm{~Hz}$ vibration of $2.5 \mu \mathrm{m}$ amplitude at $D=55 \mathrm{~cm}$.

with high SNR. Similarly, for diffusive surfaces, $w_{G}$ may need to be boosted as compared to $w_{C}$ as in such a case, SM signals with high SNR may be more difficult to obtain, contrary to SM signals with low $C$ values.

Table 1 summarizes the performances of the proposed ALL autofocus system for different surface coatings (SP: sandpaper, W: white paper, S: steel, and O: Oralite 5300) (where $\mu_{r m s}$ denotes the mean displacement RMS error obtained for 10 measurements and $\sigma$ the corresponding standard deviation from the mean RMS error ). It confirms that to obtain the same SM shape, $w_{G} / w_{C}$ should be set low for highly reflective surface and high for diffusive ones. For instance, the best results for a sand paper target at $D=55 \mathrm{~cm}$ are obtained for $w_{G} / w_{C}=8$, while it is 3 for a steel target (see media 4) and only $1 / 16$ for Oralite reflective films. Thus, $w_{G} / w_{C}$ ratio has been varied by a factor of 128. It thus underlines the strong dependence of optimal $w_{G} / w_{C}$ ratio on the nature of target surface.

\subsection{Laser-Target distance influence}

Further, as shown in eq. 3, the $C$ factor also depends on the laser-target distance. As a result, the optimal $w_{G} / w_{C}$ value is again affected. Table 2 summarizes and compares the reconstructed displacement $D_{r}$ results obtained for different $D$ and target surfaces. Note that the measured precision is approximately the same for different setups and is better than $90 \mathrm{~nm}$ except for the SP coating because in this case, the SM SNR was too low to detect all the fringes for $D>60 \mathrm{~cm}$.

doi: http://dx.doi.org/10.1109/ICSENS.2015.7370375 (C) 2015 IEEE 
(C) 2015 IEEE Personal use of this material is permitted. Permission from IEEE must be obtained for all other users, including reprinting/ republishing this material for advertising or promotional purposes, creating new collective works for resale or redistribution to servers or lists, or reuse of any copyrighted components of this work in other works

Table 1: Mean RMS Error $\mu_{r m s}$ and $\sigma$ the corresponding standard deviation based on 10 measurements each of $D_{r}$ as Compared with a PZT Reference Sensor at $D=50 \mathrm{~cm}$ with different targets (SP: sand paper, W: white paper, S: steel, O: Oralite 5300) for varying weighting. $D_{P Z T}=2.5 \mu \mathrm{m}$.

\begin{tabular}{|c|c|ccccc|}
\hline \multirow{3}{*}{ SP } & $w_{G} / w_{C}$ & - & 2 & 4 & 8 & 16 \\
\cline { 2 - 7 } & $\mu_{r m s}[\mathrm{~nm}]$ & - & 806 & 73 & 57.8 & 59 \\
\cline { 2 - 7 } & $\sigma[\mathrm{nm}]$ & - & 766 & 16 & 5.6 & 6.2 \\
\hline \hline \multirow{3}{*}{$\mathrm{W}$} & $w_{G} / w_{C}$ & 1 & 2 & 4 & 8 & 16 \\
\cline { 2 - 7 } & $\mu_{r m s}[\mathrm{~nm}]$ & 211 & 269 & 47.5 & 60.6 & 49.6 \\
\cline { 2 - 7 } & $\sigma[\mathrm{nm}]$ & 240 & 289 & 7.1 & 12.6 & 6.3 \\
\hline \hline \multirow{3}{*}{$\mathrm{S}$} & $w_{G} / w_{C}$ & 1 & 2 & 3 & 4 & 8 \\
\cline { 2 - 7 } & $\mu_{r m s}[\mathrm{~nm}]$ & 123 & 65 & 53 & 66 & 71.6 \\
\cline { 2 - 7 } & $\sigma[\mathrm{nm}]$ & 73 & 9.4 & 4.3 & 21.5 & 9.7 \\
\hline \hline \multirow{3}{*}{$\mathrm{O}$} & $w_{G} / w_{C}$ & $1 / 32$ & $1 / 16$ & $1 / 14$ & $1 / 12$ & $1 / 4$ \\
\cline { 2 - 7 } & $\mu_{r m s}[\mathrm{~nm}]$ & 97 & 68 & 103 & 117 & 579 \\
\cline { 2 - 7 } & $\sigma[\mathrm{nm}]$ & 32 & 11.8 & 47 & 35.3 & 444 \\
\hline
\end{tabular}

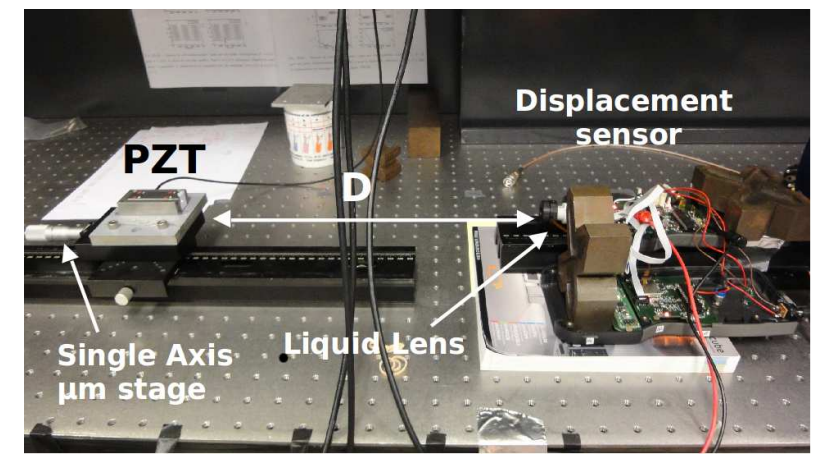

Figure 15: Photograph of the modified experimental set-up used to measure the maximum positive and negative target displacement span after having achieved feedback regime locking by using the ALL autofocus system.

As expected, for short distance $D$, the $w_{G} / w_{C}$ ratio should be reduced so that the algorithm focuses mainly on finding low $C$ value (as a higher $C$ value results in poorer precision for $\mathrm{CSU}$, as seen in Fig. 6). On the other hand for long distance $D$, the $w_{G} / w_{C}$ ratio should be increased so that the algorithm can choose the SM signals with the best amplitudes.

doi: http://dx.doi.org/10.1109/ICSENS.2015.7370375 (c) 2015 IEEE 
(C) 2015 IEEE Personal use of this material is permitted. Permission from IEEE must be obtained for all other users, including reprinting/ republishing this material for advertising or promotional purposes, creating new collective works for resale or redistribution to servers or lists, or reuse of any copyrighted components of this work in other works

Table 2: RMS Error $\epsilon_{r m s}$ of $D_{r}$ as Compared with a PZT Reference Sensor for a given distance $(D)$ and different Coatings: $\mathrm{W}$ (white paper), $\mathrm{S}$ (steel), O (Oralite 5300) and SP (sandpaper). $D_{P Z T}=2.5 \mu \mathrm{m}$.

\begin{tabular}{|c|c|ccccc|}
\hline \multicolumn{2}{|c|}{ Distance $[\mathrm{cm}]$} & 15 & 35 & 55 & 75 & 90 \\
\hline \hline \multirow{2}{*}{$\mathrm{SP}$} & $w_{G} / w_{C}$ & 8 & 8 & 8 & 8 & 8 \\
\cline { 2 - 7 } & $\mu_{r m s}[\mathrm{~nm}]$ & 47.4 & 49.1 & 52.1 & $* 1$ & $* 1$ \\
\hline \hline \multirow{2}{*}{$\mathrm{W}$} & $w_{G} / w_{C}$ & 4 & 4 & 4 & 8 & 8 \\
\cline { 2 - 7 } & $\mu_{r m s}[\mathrm{~nm}]$ & 50.1 & 52.3 & 66.9 & 61.8 & 55 \\
\hline \hline \multirow{2}{*}{$\mathrm{S}$} & $w_{G} / w_{C}$ & 0.5 & 1 & 2 & 3 & 3 \\
\cline { 2 - 7 } & $\mu_{r m s}[\mathrm{~nm}]$ & 54.8 & 59.3 & 58.6 & 69.2 & 63.8 \\
\hline \hline \multirow{3}{*}{$\mathrm{O}$} & $w_{G} / w_{C}$ & $1 / 16$ & $1 / 16$ & $1 / 16$ & $1 / 16$ & $1 / 16$ \\
\cline { 2 - 7 } & $\mu_{r m s}[\mathrm{~nm}]$ & $* 2$ & 60.7 & 63.6 & 58 & 51.6 \\
\hline
\end{tabular}

\footnotetext{
1 The SM signal SNR becomes poor causing incorrect $C$ measurement

2 Saturation of the analog SM normalization loop circuit
}

\subsection{Autofocus performances}

As it was previously mentioned, even if the $C$ value is estimated in real-time, the autofocus procedure is currently only performed once at the beginning when the target is at the initial distance $D=D_{0}$. Therefore, it is necessary to assess the maximum target displacement span $\pm \Delta$ around $D_{0}$ that allows correct displacement reconstructions. As a consequence, the experimental set-up was slightly modified by mounting the PZT on top of a single axis $\mu \mathrm{m}$ stage as shown in Fig. 15, For a given $D_{0}$, after achieving optical feedback regime locking by our proposed autofocus, the vibrating PZT is displaced by a step of $500 \mu \mathrm{m}$. Then, the precision of the vibration measurement at this new position is calculated. If it is $<\lambda / 8$ then the PZT is again displaced, and the measurement is redone, and the precision is recalculated. In this way, the procedure is repeated until the precision becomes $>\lambda / 8$. Then, the procedure is stopped and the attained total displacement span of $D_{0}+$ $N \times 500 \mu \mathrm{m}$ is noted down as the maximum achievable displacement span in a given direction for a given initial $D_{0}$ and target surface (where $N$ is the number of times PZT has been displaced). The procedure is then repeated in the other direction as well to provide both $+\Delta$ and $-\Delta$ spans.

Table 3 summarizes the results obtained for different target coatings and initial distances $D_{0}$. Note that except for highly reflective targets (Oralite here), the resulting span is usually much larger than $500 \mu \mathrm{m}$ which is sufficient for most vibration sensing measurements seeking a precision of $<\lambda / 8$. (For the specific case of Oralite, multiple phenomena disturbing the span

doi: http://dx.doi.org/10.1109/ICSENS.2015.7370375 (C) 2015 IEEE 
(C) 2015 IEEE Personal use of this material is permitted. Permission from IEEE must be obtained for all other users, including reprinting/ republishing this material for advertising or promotional purposes, creating new collective works for resale or redistribution to servers or lists, or reuse of any copyrighted components of this work in other works

Table 3: Positive and Negative target displacement span after achieving optical feedback regime locking for different Sensor-Target distances $D$ and for Mean RMS Error $\mu_{r m s}<80 \mathrm{~nm}$ (SP: sand paper, W: white paper, S: steel, O: Oralite 5300 for varying weightings). $D_{P Z T}=2.5 \mu \mathrm{m}$.

\begin{tabular}{|c|c|ccccc|}
\hline \multicolumn{2}{|c|}{ Distance $[\mathrm{cm}]$} & $15 \mathrm{~cm}$ & $35 \mathrm{~cm}$ & $55 \mathrm{~cm}$ & $75 \mathrm{~cm}$ & $90 \mathrm{~cm}$ \\
\hline \hline \multirow{3}{*}{$\mathrm{SP}$} & $w_{G} / w_{C}$ & 8 & 8 & 8 & 8 & 8 \\
\cline { 2 - 7 } & $-\Delta[\mathrm{mm}]$ & 2.5 & 4.5 & 3.5 & $* 1$ & $* 1$ \\
\cline { 2 - 7 } & $+\Delta[\mathrm{mm}]$ & 1 & 4 & 4 & $* 1$ & $* 1$ \\
\hline \hline \multirow{3}{*}{$\mathrm{W}$} & $w_{G} / w_{C}$ & 4 & 4 & 4 & 8 & 8 \\
\cline { 2 - 7 } & $-\Delta[\mathrm{mm}]$ & 2.5 & 6.5 & 2.5 & 8 & 4 \\
\cline { 2 - 7 } & $+\Delta[\mathrm{mm}]$ & 5 & 4.5 & 14.5 & 14.5 & 17 \\
\hline \hline \multirow{3}{*}{$\mathrm{S}$} & $w_{G} / w_{C}$ & 0.5 & 1 & 2 & 3 & 3 \\
\cline { 2 - 7 } & $-\Delta[\mathrm{mm}]$ & 2 & 5.5 & 1 & 6 & 5 \\
\cline { 2 - 7 } & $+\Delta[\mathrm{mm}]$ & 0.5 & 1 & 11 & 6.5 & 24 \\
\hline \hline \multirow{3}{*}{$\mathrm{O}$} & $w_{G} / w_{C}$ & $1 / 16$ & $1 / 16$ & $1 / 16$ & $1 / 16$ & $1 / 16$ \\
\cline { 2 - 7 } & $-\Delta[\mathrm{mm}]$ & $* 2$ & 0.3 & 2 & 0.5 & $* 3$ \\
\cline { 2 - 7 } & $+\Delta[\mathrm{mm}]$ & $* 2$ & 1.5 & 1 & 3 & $* 3$ \\
\hline
\end{tabular}

\footnotetext{
1 The SM signal SNR becomes poor causing incorrect $C$ measurement

2 Saturation of the analog SM normalization loop circuit

${ }^{3} \mathrm{LD}$ operating in bi-mode regime
}

measurements are cited in the footnotes of Table 3. Also note that for $D_{0}=$ $35 \mathrm{~cm}$, only a step of $300 \mu \mathrm{m}$ was achievable for the $-\Delta$ direction.)

The difference in the $\pm \Delta$ span values of Table 3 is due to the fact that the target is not necessarily at the optical focus point of the liquid lens as $f_{L L}$ is set in accordance with the measured $C$ and Gain values. It is this asymmetry which reduces the maximum possible span around the target's initial position.

In the light of these experiments, it has been demonstrated that the proposed system can perform autonomous autofocus positioning (resulting in desired SM feedback regime locking) for various targets and at different distances. In addition, even though SM displacement reconstruction is not presently done simultaneously with autofocusing, yet the tabulated results indicate that only one initial autofocus is sufficient for most applications seeking a precision of $<\lambda / 8$.

doi: http://dx.doi.org/10.1109/ICSENS.2015.7370375 (C) 2015 IEEE 
(C) 2015 IEEE Personal use of this material is permitted. Permission from IEEE must be obtained for all other users, including reprinting/ republishing this material for advertising or promotional purposes, creating new collective works for resale or redistribution to servers or lists, or reuse of any copyrighted components of this work in other works

\section{Discussion and Conclusion}

In this paper, we have demonstrated for a SM laser micro-interferometer the feasibility of an auto-adaptive optical element (here in the form of a voltage controlled liquid lens) for automatically maintaining the SM phenomenon in the moderate feedback regime. We have shown that performing SM normalization prior to any kind of processing allows not only to use CSU algorithm to reconstruct the target displacement but also to retrieve an estimation of the value of $C$. We proposed an algorithm to assess $C$ in real-time even with limited computing resources. Combining this estimated value of $C$ with the measured SM signal amplitude is the corner stone for a robust SM autofocus algorithm. It was shown that it can work with different target coatings at different distances. It results in a totally autonomous sensing system which achieves a real-time precision of less than $90 \mathrm{~nm}(\lambda / 8)$, without requiring the intervention of any SM skilled operator.

In addition, it was shown that the best results are obtained by setting $w_{G} / w_{C}$ ratio in accordance with the target surface coating and sensor to target distance. The higher the sensor to target distance is and the more diffusive the surface coating is, the higher this ratio should be. This paper also shows that both $C$ and $A_{S M}$ are necessary to robustly perform an autofocus on a wide variety of surfaces. It was clearly demonstrated that SM displacement reconstruction cannot be correctly performed if the autofocus is only based on seeking higher $A_{S M}$ in particular for reflective targets as it might result in: (1) saturation of the analog front-end circuit, (2) strong feedback $C$ factor and/or (3) bi-mode behavior of the LD.

Further, since currently the autofocus algorithm itself is only performed once, it was verified that maximum target displacement span after having locked to moderate feedback regime was still acceptable for most vibration measurements seeking a precision of $<\lambda / 8$ for different target surfaces as well as distances.

Finally, the following improvements can be incorporated in an optimization algorithm that:

- could try to tune around $V_{b 1}$ in order to obtain a specific predefined $C$ value once a good liquid lens position is found

- could take advantage of the availability of real-time measurements of $C$ and Gain in order to perform real-time adjustments of the liquid lens position.

- could also take into account the currently noticed asymmetry in the maximum displacement span and adjust the adaptive element to reduce

doi: http://dx.doi.org/10.1109/ICSENS.2015.7370375 (C) 2015 IEEE 
(c) 2015 IEEE Personal use of this material is permitted. Permission from IEEE must be obtained for all other users, including reprinting/ republishing this material for advertising or promotional purposes, creating new collective works for resale or redistribution to servers or lists, or reuse of any copyrighted components of this work in other works

it.

\section{Acknowledgment}

The authors acknowledge the funding of CALDIRO ANR Emergence and the technical support provided by F. Jayat and L.E. Maneville.

\section{References}

[1] S. Donati, "Developing self-mixing interferometry for instrumentation and measurements," Laser \& Photon. Rev., vol. 6, pp. 393417, 2012.

[2] S. Donati, M. Norgia, "Self-Mixing Interferometry for Biomedical Signals Sensing," Selected Topics in Quantum Electronics, IEEE Journal of , vol.20, no.2, pp. 104-111, March-April 2014.

[3] O. D. Bernal, U. Zabit, and T. Bosch, "Classification of laser self-mixing interferometric signal under moderate feedback," Appl. Opt. 53, pp. 702$708,2014$.

[4] G. Plantier, C. Bes, and T. Bosch, "Behavioral model of a self-mixing laser diode sensor," Quantum Electronics, IEEE Journal of, vol. 41, no. 9, pp. 1157-1167, 2005.

[5] T. Taimre and A. D. Rakić, "On the nature of Ackets characteristic parameter C in semiconductor lasers," Appl. Opt. 53, pp. 1001-1006, 2014.

[6] C. Bes, V. Belloeil, G. Plantier, Y. Gourinat, and T. Bosch, "A selfmixing laser sensor design with an extended kalman filter for optimal online structural analysis and damping evaluation", Mechatronics, IEEE/ASME Transactions on, vol. 12, no. 3, pp. 387394, 2007.

[7] Y. Yanguang, X. Jiangtao, J.F. Chicharo, T. Bosch, "Optical Feedback Self-Mixing Interferometry With a Large Feedback Factor C : Behavior Studies," Quantum Electronics, IEEE Journal of , vol.45, no.7, pp. 840848 , July 2009

[8] A. Magnani, A. Pesatori, and M. Norgia, "Self-mixing vibrometer with real-time digital signal elaboration," Appl. Opt. 51, pp. 5318-5325, 2012.

doi: http://dx.doi.org/10.1109/ICSENS.2015.7370375 (c) 2015 IEEE 
(c) 2015 IEEE Personal use of this material is permitted. Permission from IEEE must be obtained for all other users, including reprinting/ republishing this material for advertising or promotional purposes, creating new collective works for resale or redistribution to servers or lists, or reuse of any copyrighted components of this work in other works

[9] Y. Fan, Y. Yu, J. Xi, and J. F. Chicharo, "Improving the measurement performance for a self-mixing interferometry-based displacement sensing system," Appl. Opt. 50, pp. 5064-5072, 2011.

[10] G. Giuliani, S. Bozzi-Pietra and S. Donati "Self-mixing laser diode vibrometer", Meas. Sci. Technol. 14 24-32, 2003.

[11] U. Zabit, O. Bernal, T. Bosch "Design and analysis of an embedded accelerometer coupled Self-Mixing laser displacement sensor" IEEE Sensors journal, Vol.13, N6, pp. 2200-2207, 2013.

[12] C. Bes, G. Plantier and T. Bosch, "Displacement measurements using a self-mixing laser diode under moderate feedback", IEEE Trans. Instrum. and Meas. Vol. 55, pp. 1101-1105, 2006.

[13] Zabit, U.; Bernal, O.D.; Bosch, T., "Self-mixing sensor for real-time measurement of harmonic and arbitrary displacements," Instrumentation and Measurement Technology Conference (I2MTC), $201 \overline{2 \text { IEEE Interna- }}$ tional , pp. 754,758, 13-16 May 2012.

[14] U. Zabit, R. Atashkhooei, T. Bosch, S. Royo, F. Bony and A. D. Rakić, "Adaptive self-mixing vibrometer based on a liquid lens", Optics Letters, Vol. 35 Issue 8, pp. 1278-1280 , 2010.

[15] Atashkhooei, R.; Royo, S.; Azcona, F.J., "Dealing With Speckle Effects in Self-Mixing Interferometry Measurements," Sensors Journal, IEEE , vol.13, no.5, pp. 1641-1647, 2013.

[16] S. Merlo and S. Donati, "Reconstruction of displacement waveforms with a single-channel laser-diode feedback interferometer", Quantum Electronics, IEEE Journal of, vol.33, no.4, pp.527,531, Apr 1997.

[17] R. Lang and K. Kobayashi, "External optical feedback effects on semiconductor injection laser properties," IEEE J. Quantum Electron., vol. 16, no. 3, pp. 347-355, 1980.

[18] K. Petermann, "External optical feedback phenomena in semiconductor lasers," IEEE J. Sel. Topics Quantum Electron., vol. 1, no. 2, pp. 480-489, 1995.

[19] G. Giuliani and S. Donati, "Unlocking Dynamical Diversity - optical feedback effects on semiconductors lasers", D. M. Cane and K. A. Shore, Eds. John Wiley \& Sons, Ltd, 2005.

doi: http://dx.doi.org/10.1109/ICSENS.2015.7370375 (c) 2015 IEEE 
[20] O.D. Bernal, U. Zabit, and T. Bosch, "Study of laser feedback phase under self-mixing leading to improved phase unwrapping for vibration sensing," Sensors Journal, IEEE, vol. 13, no. 12, pp. 4962-4971, 2013.

[21] R. Atashkhooei, S. Royo, F. Azcona, U. Zabit, "Analysis and control of speckle effects in self-mixing interferometry," Sensors, IEEE, pp. 13901393, 28-31 Oct. 2011.

[22] Giuliani, Guido and Donati, Silvano and Elsässer, Wolfgang, "Measurement of linewidth enhancement factor of different semiconductor lasers in operating conditions", Photonics Europe, pp 61841-61841, 2006. 